$63^{\text {ème }}$ Congrès de la SFCO, 02021 (2015)

DOI:10.1051/sfco/20156302021

(C) Owned by the authors, published by EDP Sciences, 2015

\title{
COMMUNICATION
}

\section{La distraction symphysaire dans la prise en charge de l'encombrement dentaire mandibulaire}

\author{
Savoldelli $\mathrm{C}^{* * * *}$, Castro $\mathrm{R}^{* * * *}$, Olympo $\mathrm{M}^{* * * *}$, Cochais $\mathrm{P}^{* *}$, Manière-Ezvan $\mathrm{A}^{* *}$ \\ * Institut Universitaire de la Face et du cou de Nice \\ ** Service de Soins Dentaires CHU de Nice
}

Le traitement de l'encombrement incisif mandibulaire associé à un déficit osseux associe habituellement des extractions dentaires et un traitement orthodontique. La distraction symphysaire a été proposée pour préserver le capital dentaire. Cette technique consiste à réaliser une ostéotomie symphysaire suivie d'une distraction transversale afin d'augmenter le périmètre d'arcade mandibulaire. Cette technique demeure encore marginale car perçue comme invasive, complexe et risquée. Nous présentons notre évaluation de cette technique afin d'en préciser les indications, les avantages et les limites, et pour proposer un protocole chirurgical simplifié.

Nom et adresse du conférencier

\section{Charles SAVOLDELLI}

Institut Universitaire de la Face et du Cou - Département de chirurgie orale

et maxillo-faciale

31 avenue de Valombrose

Nice (France)

savoldelli.c@chu-nice.fr

This is an Open Access article distributed under the terms of the Creative Commons Attribution License 4.0, which permits unrestricted use, distribution, and reproduction in any medium, provided the original work is properly cited. 